\title{
Phase Noise Investigation of Multicarrier Sub-THz Wireless Transmission System Based on an Injection-Locked Gain-Switched Laser
}

\author{
Tong Shao, Haymen Shams, Member, IEEE, Prince M. Anandarajah, Senior Member, IEEE, Martyn J. \\ Fice, Member, IEEE, Cyril C. Renaud, Member, IEEE, Frédéric van Dijk, Alwyn J. Seeds, Fellow, \\ IEEE, and Liam P. Barry, Senior Member, IEEE
}

\begin{abstract}
We propose a multi-carrier $\mathrm{THz}$ wireless communication system using an injection-locked gain-switched laser as an optical comb source. The phase noise of the $192 \mathrm{GHz}$ signal resulting from the beating of two optical comb lines is theoretically analyzed and experimentally examined. Moreover, a three channel, 10 Gbaud QPSK THz signal is generated, and transmission over $40 \mathrm{~km}$ standard single mode fiber (SSMF) is experimentally demonstrated.
\end{abstract}

Index Terms - Phase noise, gain-switched laser, optical comb, frequency multiplication

\section{INTRODUCTION}

$\mathrm{D}$ ata rates in wireless communications have been increasing exponentially over recent decades. However the spectral resources are extremely limited because of the heavy use of today's conventional frequency range up to $60 \mathrm{GHz}$. High-speed terahertz (THz) wireless communications have attracted great interest for short distance ultrahigh data rate mobile applications [1]. A photonic solution is a promising technique for high-frequency RF signal generation and transmission, as it enables the distribution of high-frequency RF signals over long distance through optical fiber, and makes the system compact and light [2].

Several systems have been demonstrated based on heterodyne detection for increasing the bit rates up to $100 \mathrm{Gbit} / \mathrm{s}$ [3-7]. A multicarrier based system with optical subcarriers was demonstrated in the W-band to maximize the overall channel data rate, and achieve high spectral efficiency [3]. The wireless transmission window in the $200 \mathrm{GHz}$ band is of strong interest due to low atmospheric transmission losses.

Manuscript received Jan. 1st, 2015. This work was supported in part by the SFI PI grant 09/IN.1/I2653 and 10/CE/I1853, the HEA PRTLI 4 INSPIRE Programs, the Engineering and Physical Sciences Research Council programme grant Coherent Terahertz Systems (COTS) (EP/J017671/1), and by the European Commission through the European project iPHOS (grant agreement no: 257539).

T. Shao, P.M. Anandarajah, and L.P. Barry are with Radio and Optical Communication Lab, Rince Institute, Dublin City University, Dublin 9, Ireland.

H. Shams, M.J. Fice, C.C. Renaud and A.J. Seeds are with Department of Electronic and Electrical Engineering, University College London, UK.

F. van Dijk is with III-V Lab, a joint Laboratory of "Alcatel Lucent Bell Labs", "Thales Research \& Technology" and "CEA-LETI", France.
Recently $75 \mathrm{Gbit} / \mathrm{s}$ multichannel transmission at $200 \mathrm{GHz}$ carrier frequency using two free running lasers and a digital coherent receiver has been experimentally demonstrated $[4,5]$. However, the frequency spacing between two lasers is not constant and their phases fluctuate continuously. To stabilize the carrier frequencies, an optical frequency comb-based signal generation is the most effective approach [6-8]. A. Kanno et al. [6] and T. Nagatsuma et al. [7] have experimentally demonstrated 16 quadrature amplitude modulation (QAM) and quadrature phase shift keying (QPSK) systems in the W-band using optical frequency comb-based signal generation. Both of the optical combs used in these works were based on external modulation. The large insertion loss of the multiple cascaded modulators, coupled with the modulation efficiency and the instability induced by bias drift can prove prohibitive for broader optical comb generation. In [8], a $100 \mathrm{~Gb} / \mathrm{s} \mathrm{THz} \mathrm{system}$ using a mode-locked laser (MLL) as an optical comb source is proposed. Although this technique can generate multi-carrier signals spanning over a wide bandwidth, it inherently suffers from cavity complexity due to the use of a MLL, and does not offer free spectral range (FSR) tunability since the comb line spacing is fixed by the cavity length of the laser.

Previously, we reported on the use of gain-switching to generate an optical comb [9]. Such a comb source enables simple and cost efficient generation of lightwaves with precisely controlled channel spacing. Different $60 \mathrm{GHz}$ radio over fiber (RoF) systems using the gain-switched comb source have been proposed and demonstrated [10,11], with the data rate limited to $25 \mathrm{~Gb} / \mathrm{s}$ due to the limited bandwidth of the $60 \mathrm{GHz}$ technology [11].

In this paper, we propose a multi-carrier $\mathrm{THz}$ wireless transmission system using the externally injected gain-switched laser as an optical comb source. The phase noise of the $192 \mathrm{GHz}$ signal resulting from the beating of two optical comb lines is theoretically analyzed and experimentally examined. Moreover, three 10 Gbaud QPSK sub-THz channel signals (with total data rate of $60 \mathrm{Gbit} / \mathrm{s}$ ) are generated and transmitted over $40 \mathrm{~km}$ standard single mode fiber (SSMF) before wireless transmission.

The article is organized as follows. In section II, the principle of $\mathrm{THz}$ generation using a gain-switched comb source is explained and the phase noise of the $\mathrm{THz}$ signal generated by 


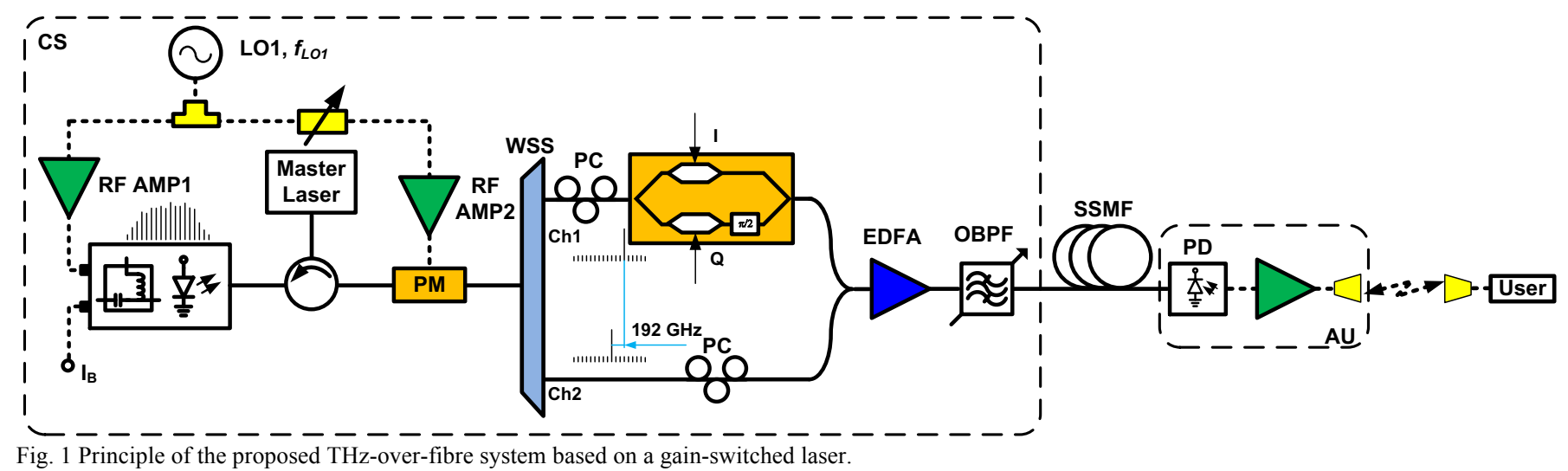

the photonic-RF frequency multiplication is theoretically analyzed and experimentally demonstrated. In section III, a multi-carrier wireless communications $\mathrm{THz}$ system based on the gain-switched comb source is experimentally demonstrated. Finally our conclusions are presented in section IV.

\section{PRinciple of THz System And InVEStigation of Phase NOISE}

In this part, we will present the principle of the proposed $\mathrm{THz}$ signal generation and transmission system using a gain-switched laser as an optical comb source. Then the phase noise of the THz signal resulting from the beating of two optical tones of the gain-switched laser is theoretically analyzed and experimentally demonstrated.

\section{A. Principle of the THz system based on gain-switched laser}

Fig. 1 shows the proposed $\mathrm{THz}$ multi-carrier transmission system employing the gain-switched laser. A distributed feedback (DFB) laser is used to generate a comb by gain switching the laser. A master laser is used for external injection into the gain switched laser in order to reduce the linewidth of optical comb lines and mitigate the chirp [9]. Moreover, the injection locking can enhance the relaxation oscillation frequency of the slave laser and thus improve the flatness of the optical comb [9]. An optical phase modulator is employed to broaden the spectrum of the optical comb. The spacing between the subcarriers is controlled by the driving RF frequency. A wavelength selective switch (WSS) is used to select two comb lines, into two different optical channels. One optical tone in channel 1 (Ch1) is fed into a dual parallel Mach-Zehnder modulator (DP-MZM) and modulated with a QPSK signal. The other optical tone in channel 2 (Ch2) is used as an optical local oscillator (LO) for $\mathrm{THz}$ signal generation. The recombined signal is then amplified with an EDFA and transmitted over standard single-mode fiber (SSMF) to the antenna unit (AU).

At the AU, the optical LO source beats with the modulated optical signal on an unpackaged uni-travelling carrier (UTC) photodiode [12] to generate the $\mathrm{THz}$ modulated multichannel signal. The modulated $\mathrm{THz}$ signal is radiated to the end user through a pair of horn antennas.

\section{$B$. Investigation of the phase noise}

The phase noise performance of the THz signal generated by the beating of two optical comb lines is firstly examined. The free spectral range (FSR) of the optical comb is initially set at $16 \mathrm{GHz}$. Two optical tones with the frequency spacing of $192 \mathrm{GHz}$ are selected into two optical channels of the WSS. The QPSK data is not applied to the DP-MZM in order to initially measure the phase noise of the resultant $192 \mathrm{GHz}$ signal. The $192 \mathrm{GHz}$ signal is down-converted to an intermediate frequency (IF) using a sub-harmonic mixer. The LO signal at $17 \mathrm{GHz}$, from a RF synthesizer, is firstly frequency multiplied by using a sixth harmonic electronic multiplier and then applied to the sub-harmonic mixer to down-convert the resultant $192 \mathrm{GHz}$ signal to $12 \mathrm{GHz}$.

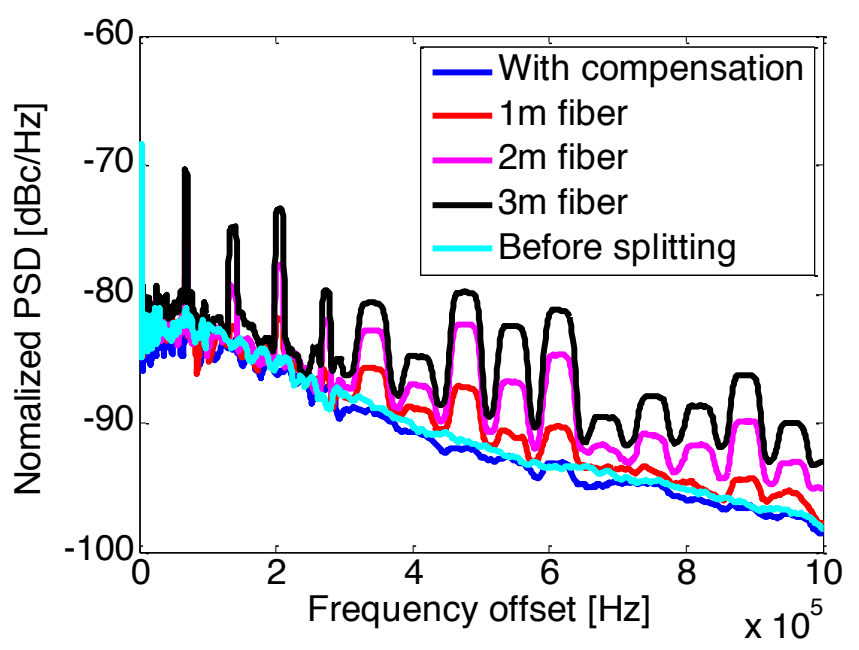

Fig. 2 Phase noise measurement of the resultant $192 \mathrm{GHz}$ signal.

We have previously demonstrated that a time delay between the two optical channels will induce significant phase noise due to the phase decorrelation of the two optical tones [10]. Therefore the delay between the two path lengths is accurately compensated with the study of the spectrum of the resultant $\mathrm{THz}$ signal [10]. Additional $1 \mathrm{~m}, 2 \mathrm{~m}$ or $3 \mathrm{~m}$ delay fibers are applied to $\mathrm{Ch} 2$ to examine the phase noise impact due to the optical phase decorrelation. Fig. 2 shows phase noise measurements of the down-converted $\mathrm{THz}$ signal. In order to examine the delay compensation, the phase noise of the $192 \mathrm{GHz}$ signal generated by the beating of the two optical tones without splitting is also measured. In this case, both of the two optical tones with $192 \mathrm{GHz}$ frequency spacing are selected by one port of the WSS. Therefore, no additional optical delay is applied between the two optical tones. 
From Fig. 2, it can be seen that the phase noise of the $192 \mathrm{GHz}$ signal is increasing as the time delay between the two optical channels is increased. Comparing the cyan line and the blue line in Fig. 2, the phase noise performance of the $\mathrm{THz}$ signal with delay compensation and without optical splitting is the same. Thus it is evident that the phase noise induced by the optical phase decorrelation can be highly mitigated with the delay compensation technique.

A 12.5 Gbaud QPSK signal is then applied to the DP-MZM. Fig. 3 (a), (c) and (e) show the constellations and error vector magnitude (EVM) of the $192 \mathrm{GHz}$ QPSK signal with different levels of phase noise due to the phase decorrelation by varying the time delay between the two channels. It is important to note that there is no phase correction applied in the DSP. It is evident that the compensation of the time delay between the two channels can partially reduce the phase noise impact. Nevertheless Fig. 3 (a) also shows that the $192 \mathrm{GHz}$ QPSK signal still suffers from some level of phase noise even though the optical delay is fully compensated. Fig. 3 (b), (d) and (f) show constellations of the QPSK signal with different fiber delay where digital phase estimation is applied in the DSP process to mitigate the phase noise impact [13].
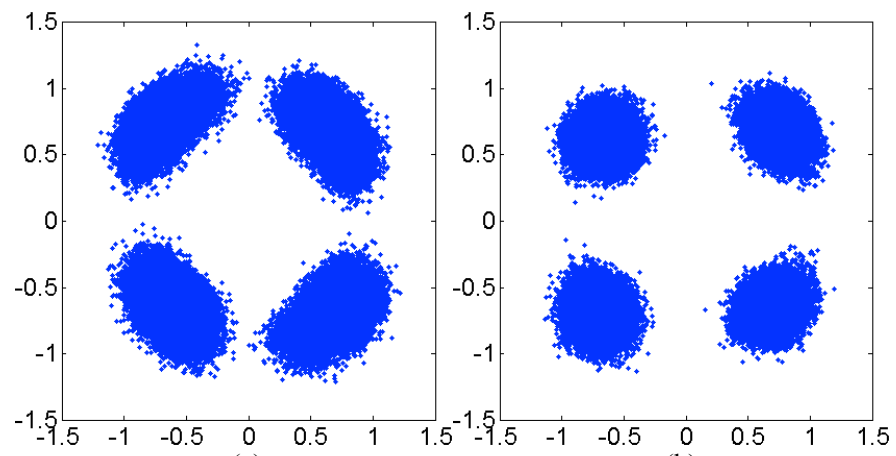

(a)

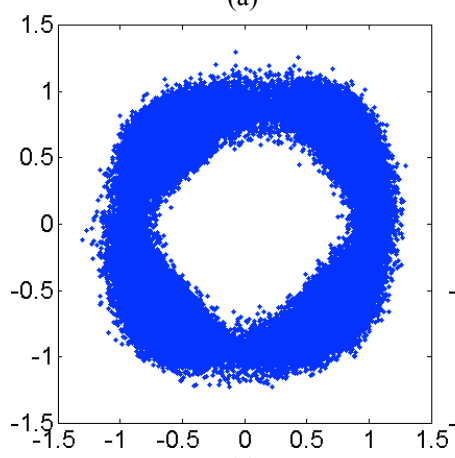

(b)

(c)

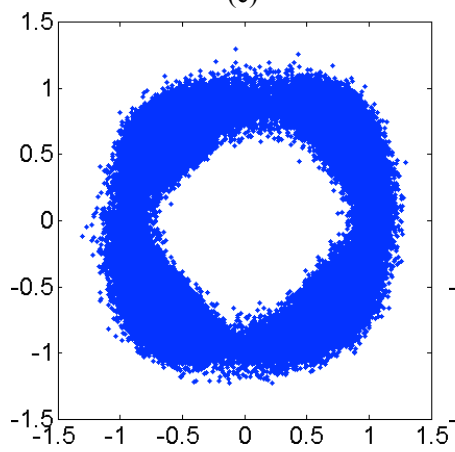

(e)

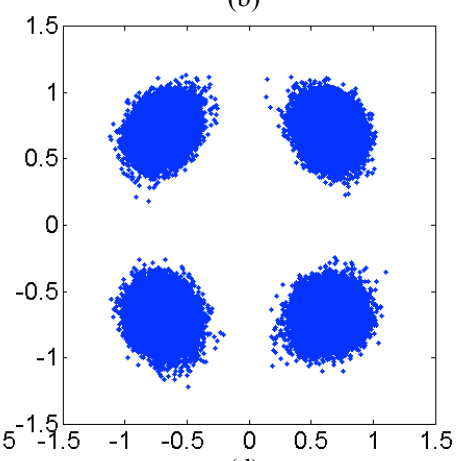

(d)

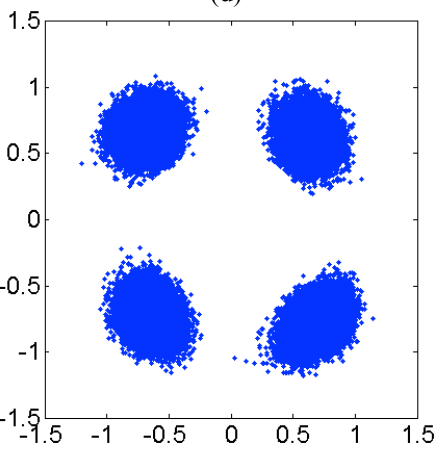

(f)
Fig. 3 Constellations of the $192 \mathrm{GHz}$ QPSK signal with different delay. (a) with delay compensation, without DSP phase correction, $\mathrm{EVM}=19.1 \%$ (b) with delay compensation, with DSP phase correction, $\mathrm{EVM}=14.6 \%$ (c) $1 \mathrm{~m}$ fiber delay, without DSP phase correction, $\mathrm{EVM}=20 \%$, (d) $1 \mathrm{~m}$ fiber delay, with DSP phase correction, $\mathrm{EVM}=14.3 \%$ (e) $3 \mathrm{~m}$ fiber delay, without DSP phase correction, $\mathrm{EVM}=22.4 \%$ (f) $3 \mathrm{~m}$ fiber delay, with DSP phase correction, $\mathrm{EVM}=14.5 \%$.

It can be seen from the constellation shown in Fig. 3 (a) that there is some level of phase noise impact even though the optical delay is fully compensated. Previously, we have demonstrated a $60 \mathrm{GHz}$ RoF system employing a high-linewidth gain-switched laser [11]. It has been proven that the phase noise impact can be highly mitigated by compensating the time delay between the two channels even if a high-linewidth $(60 \mathrm{MHz})$ gain-switched laser without external injection is employed in the system. Compared to the $60 \mathrm{GHz}$ system, the proposed $\mathrm{THz}$ system using the gain-switched comb source is based on a higher-order photonic-RF frequency multiplication. The output of the gain-switched laser can be expressed as:

$$
E_{G S}(t)=I\left(I_{D C}+E_{L O 1}(t)\right) \exp \left(j 2 \quad f_{0} t+{ }_{0}(t)\right)
$$

where $E_{G S}(t)$ is the output of the gain-switched laser. $I(\bullet)$ represents the relationship between bias current of the laser with the amplitude of the optical output. $I_{D C}$ is the DC bias of the gain-switched laser. $f_{0}$ and $\phi_{0}(t)$ are the central frequency and phase noise of the master laser respectively as the gain-switched laser is injection-locked by the master laser. $E_{L O I}(t)$ is the electrical field of the LO1, which can be expressed as:

$$
E_{L O 1}(t)=I_{L O 1} \cos \left(2 f_{L O 1} t+{ }_{L O 1}(t)\right)
$$

where $f_{L O I}$ and $\phi_{L O I}(t)$ present the central frequency and the phase noise of the LO1 signal respectively, $I_{L O I}$ is the amplitude of the LO1 signal. The optical field of the gain-switched laser in equation (1) can be derived using Taylor series:

$$
\begin{aligned}
& E_{G S}(t)=\exp \left(j 2 f_{0} t+{ }_{0}(t)\right)\left[A_{0}+\right. \\
& A_{1} E_{L O 1}(t)+A_{2} E_{L O 1}^{2}(t)+\ldots+A_{n} E_{L O 1}^{n}(t)+\ldots
\end{aligned}
$$

where $A_{0}, A_{1}, \ldots A_{n}, \ldots$ are constant. Assuming that the optical power of the gain-switched laser is mainly distributed over 11 optical lines, equation (3) can be rewritten as:

$$
\begin{aligned}
& E_{G S}(t)=\exp \left(j 2 f_{0} t+{ }_{0}(t)\right)\left[B_{0}+\right.
\end{aligned}
$$

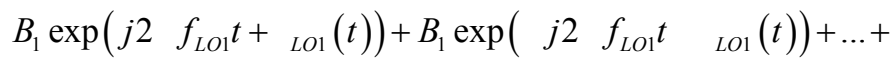

$$
\begin{aligned}
& B_{5} \exp \left(j 10 f_{L O 1} t+5_{L O 1}(t)\right)+B_{5} \exp \left(\begin{array}{llll}
j 10 & f_{L O 1} t & 5_{L O 1}(t)
\end{array}\right)
\end{aligned}
$$

where $B_{0}, B_{1}, \ldots, B_{5}$ are constants. The output of the gain-switched laser is sent to an optical phase modulator for spectral broadening. The optical comb at the output of the phase modulator can be thus expressed as: 


$$
E_{\text {comb }}(t) \quad E_{G S}(t) \exp j \frac{E_{L O 1}(t)}{V}
$$

By using Jacobi-Anger expansion, equation (5) can be derived as:

$$
E_{\text {comb }}(t) \quad E_{G S}(t)_{n=}^{+} j^{n} J_{n}\left(I_{L O 1}\right) \exp \left(j n\left(2 f_{L O 1} t+{ }_{L O 1}(t)\right)\right)(
$$

where $J n(\bullet)$ is the $\mathrm{n}^{\text {th }}$ Bessel function. Substituting (4) into (6), the output of the optical comb containing 13 optical tones can be expressed as:

$$
\begin{aligned}
& E_{\text {comb }}(t)=\exp \left(j 2 \quad f_{0} t+{ }_{0}(t)\right)\left[C_{0}+\right. \\
& C_{1} \exp \left(j 2 f_{L O 1} t+{ }_{L O 1}(t)\right)+C_{1} \exp \left(\begin{array}{lll}
j 2 & f_{L O 1} t & \\
L O 1 & (t))+\ldots+
\end{array}\right. \\
& C_{6} \exp \left(j 12 f_{L O 1} t+6_{L O 1}(t)\right)+C_{6} \exp \left(\begin{array}{llll}
j 12 & f_{L O 1} t & \left.6_{L O 1}(t)\right)
\end{array}\right.
\end{aligned}
$$

where $C_{0}, C_{l}, \ldots, C_{6}$ are constants. In the experiment, the $\mathrm{THz}$ signal is generated by a beating of two comb lines spaced by $192 \mathrm{GHz}\left(12 \times f_{L O I}\right)$. Therefore the two optical comb lines can be expressed as:

$$
\begin{aligned}
& E_{\text {comb1 }}(t) \exp \left(j\left(2\left(f_{0}+6 f_{L O 1}\right) t+{ }_{0}(t)+6_{\text {LO1 }}(t)\right)\right) \\
& \left.E_{\text {comb2 }}(t) \exp \left(j\left(\begin{array}{lll}
2 & \left(f_{0}\right. & 6 f_{L O 1}
\end{array}\right) t+{ }_{0}(t) \quad 6_{L O 1}(t)\right)\right)
\end{aligned}
$$

The photocurrent of the UTC-PD is:

$$
\begin{aligned}
& i_{U T C P D}\left|E_{\text {comb1 }}(t)+E_{\text {comb } 2}(t)\right|^{2} \\
& =I_{D C}+2 \cos \left(24 f_{L O 1} t+12_{L O 1}(t)\right)
\end{aligned}
$$

The resultant $\mathrm{THz}$ signal is:

$$
i_{T H z} \quad \cos \left(24 f_{L O 1} t+12_{L O 1}(t)\right)
$$

More generally speaking, the phase noise of the resulting RF signal can be simply linked to the phase noise of the LO1 signal as:

$$
\text { Photonic } R F=n_{L O 1}(t)
$$

where $\phi_{\text {Photonic-RF }}(t)$ represents the phase noise of the resulting RF signal. Here we define a random phase change of the LO1 signal and resulting RF signal between $\mathrm{t}$ and $t+\tau$ as:

$$
\begin{aligned}
& L O 1()=\quad L O 1(t+) \quad L O 1(t) \\
& \text { Photonic } R F()=n \quad L O 1(t+) \quad L O 1(t)
\end{aligned}
$$

The variance of the random phase change between $t$ and $t+\tau$ of the LO signal $\left(\sigma_{\Delta \phi L O}{ }^{2}(\tau)\right)$ is related to the PSD of the instantaneous angular frequency fluctuation $S_{f}(\omega)$ of the LO1 signal [14]

$$
{ }_{L O 1}^{2}()=\frac{2}{2}+\frac{\sin \frac{2}{2}}{2} S_{f}() d
$$

The instantaneous frequency fluctuation here is considered as white noise, which means the PSD of the frequency fluctuation is a constant $\left(S_{f}(\omega)=C\right)$, the variance of the random phase change between $\tau$ delay $\left(\sigma_{\triangle \phi L O}{ }^{2}(\tau)\right)$ is represented as

$$
{ }_{20}^{2}()=2_{L O}||
$$

where $2 \gamma_{L O I}$ are the angular full linewidth of the LO1 signal. The variance of the random phase change between $t$ and $t+\tau$ of the resulting RF signal $\left(\sigma_{\triangle \phi P \text { Phtonic-RF }}{ }^{2}(\tau)\right)$ can be expressed as:

$$
\begin{aligned}
& 2_{\text {Photonic } R F}()=n^{2} \\
= & 2 n_{L O}^{2}()
\end{aligned}
$$

And the linewidth of the resulting RF signal can be expressed as:

$$
\text { Photonic } R F=n^{2} \quad L O 1
$$

where $2 \gamma_{\text {Photonic-RF }}$ is the angular full linewidth of the resulting $\mathrm{RF}$ signal. It can be seen from equation (10) and (11) that the $\mathrm{RF}$ signal generated by high-order frequency multiplication suffers from a higher level of phase noise induced by the LO signal. In other words, the phase correlation among the optical comb lines based on the gain-switched laser is not even. If we assume that the dominant frequency jitter component of the RF signal and LO1 signal is white noise, the normalized power spectral density (PSD) of the resulting RF signal and the LO1 signal follows the Lorentzian slope if the delay of the two channels are well compensated [15]:

$$
\begin{aligned}
& S_{L O 1}(f)=10 \log _{10} \frac{L O 1}{{ }_{L O 1}+4^{2} f^{2}} \\
& S_{\text {Photonic } R F}(f)=10 \log _{10} \frac{n^{2} L O 1}{\left(n^{2}{ }_{L O 1}\right)^{2}+4{ }^{2} f^{2}}
\end{aligned}
$$

Here it is important to note that the $\mathrm{THz}$ signal is firstly mixed with another THz signal in the sub harmonic mixer, for electrical down-conversion before sending it to the electrical spectrum analyzer for phase noise measurement. Therefore the phase noise of the down-converted signal shown in Fig. 2 contains a contribution both from LO1 and LO2 signals. Similarly the THz signal generated by $12^{\text {th }}$ order RF frequency multiplication of LO2 signal can be expressed as:

$$
i_{\mathrm{THZ}_{-} L O} \quad \cos \left(24 f_{\mathrm{LO} 2} t+12_{\mathrm{LO} 2}(t)\right)
$$

where $f_{\mathrm{LO} 2}$ and $\phi_{\mathrm{LO} 2}(t)$ present the central frequency of the LO2 signal respectively. Thus the down-converted $\mathrm{THz}$ signal can be expressed as: 


$$
i_{I F} \quad \cos \left(2 \quad\left(\begin{array}{lll}
12 f_{L O 2} & 12 f_{L O 1}
\end{array}\right) t+12\left({ }_{L O 2}(t) \quad{ }_{L O 1}\right)\right)
$$

Fig. 4 shows the normalized PSD of the $192 \mathrm{GHz}$ signal which is down-converted to $12 \mathrm{GHz}$, the $16 \mathrm{GHz}$ signal which is generated by the beating of a two neighboring tones, the electrical LO1 signal, and the electrical LO2 signal. It can be seen that the phase noise of the $192 \mathrm{GHz}$ signal is about $20 \mathrm{~dB}$ larger than the $16 \mathrm{GHz}$ signal which is a result of the beating of a pair of neighboring optical tones. It is also shown that the phase noise of the LO2 signal is smaller $(>3 \mathrm{~dB})$ than LO1 signal. It is evident that the dominant phase noise contribution of the down-converted $\mathrm{THz}$ signal is from the LO1 signal.

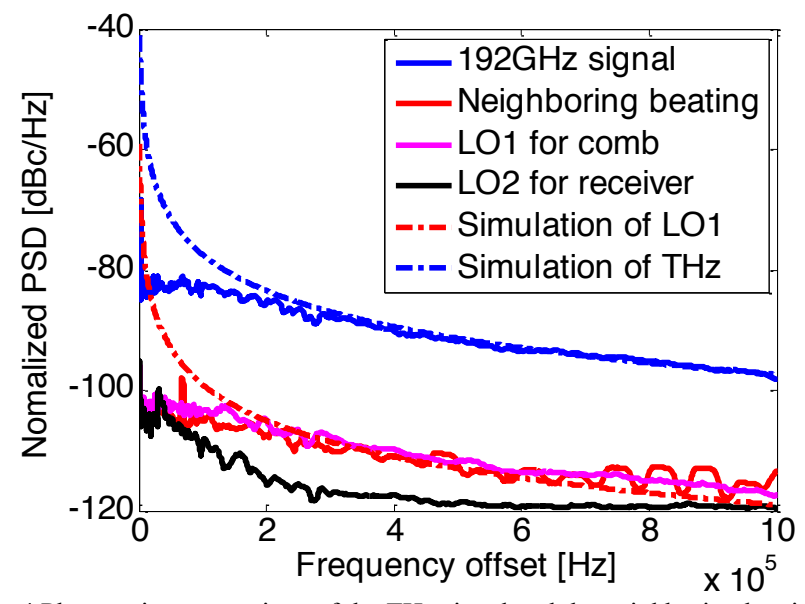

Fig. 4 Phase noise comparison of the THz signal and the neighboring beating.

A QPSK transmission with or without the impact of the phase noise due to high-order frequency multiplication is simulated. The angular linewidth of the LO1 signal $Y_{L O I}$ is set to $50 \mathrm{rad} / \mathrm{s}$, which corresponds to the simulation of the PSD of the LO1 signal. The baud rate of the QPSK signal is set to $12.5 \mathrm{Gbaud}$ and the length of the sequence is $1.2 \times 10^{5}$ symbols, which correspond to the experimental setup (experimental results are shown in Fig. 3). Signal-to-noise ratio is set to $15.5 \mathrm{~dB}$. Fig. 5 shows the constellations of the QPSK signal with or without the phase noise impact due to the high-order frequency multiplication. It can be seen that the phase noise due to high-order frequency multiplication can highly degrade the QPSK signal transmission. The EVM results of the simulation agree well with the experimental results that are shown in Fig. 3 (a) and (b).
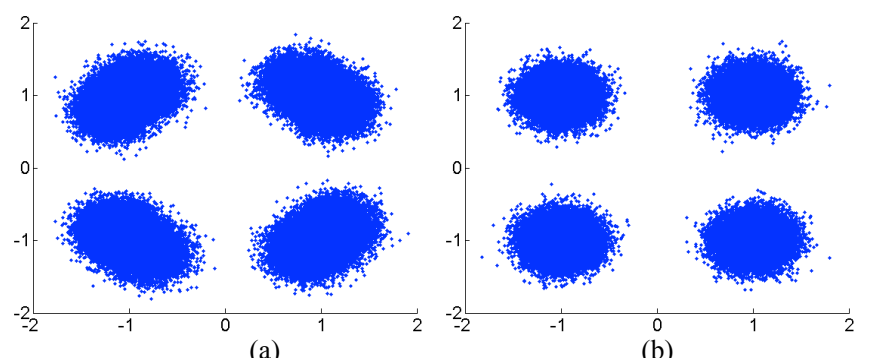

(b)

Fig.5 Constellations of the simulated QPSK signal with or without phase noise impact due to high-order frequency multiplication. (a) with phase noise impact, $\mathrm{EVM}=19.4 \%$ (b) without phase noise impact, $\mathrm{EVM}=14.6 \%$.

Based on the theoretical analysis and experimental demonstration of the phase noise of the $\mathrm{THz}$ signal, it can be concluded that:

1) The phase noise induced by the optical phase decorrelation between the two optical tones due to the time delay can be highly mitigated by delay compensation.

2) The THz signal may suffer some level of phase noise impact due to the high-order frequency multiplication even if the optical delay is compensated. This requires DSP techniques to mitigate the residual phase noise impact. However it is worthwhile to pointing out that the frequency stability and phase noise performance is much better than that of the $\mathrm{THz}$ produced by the beating of two free running lasers [4]. For example, in Ref. [4], the authors employed a laser source with $3-\mathrm{dB}$ linewidth of $15 \mathrm{kHz}$ as the transmitter and an external cavity laser (ECL) with the $3-\mathrm{dB}$ linewidth of $100 \mathrm{kHz}$ as the receiver. In this case, the linewidth of the resultant $\mathrm{THz}$ signal can be estimated around $115 \mathrm{kHz}$, while the linewidth of the resultant $\mathrm{THz}$ in our experiment is much smaller (please see Fig. 4). In our experiment, no particular frequency offset estimation is required in the DSP.

\section{EXPERIMENTAL DEMONSTRATION OF MUlti-CARRIER THZ SYSTEM BASED ON A GAIN-SWITCHED LASER}

In this section, an experimental demonstration of a multi-carrier $\mathrm{THz}$ system is presented. Three QPSK $\mathrm{THz}$ signals with a total data rate of $60 \mathrm{~Gb} / \mathrm{s}$ are generated and transmitted over $40 \mathrm{~km} \mathrm{SSMF.}$

\section{A. Experimental setup}

Fig. 6 shows the proposed $\mathrm{THz}$ multi-carrier transmission system employing the gain-switched comb source. A distributed feedback (DFB) laser at a wavelength of $1551 \mathrm{~nm}$ was used to generate a comb by gain switching the laser with the aid of a $24 \mathrm{dBm}$ RF signal. A master laser with a linewidth of $300 \mathrm{kHz}$ was used for external injection into the gain switched laser in order to reduce the linewidth of optical comb lines [9]. An optical phase modulator is employed to broaden the spectrum of the optical comb for higher frequency signal generation. After amplifying the optical signal using an Erbium-doped fiber amplifier (EDFA), a WSS is used to select two or several comb lines, into two different optical channels. The WSS employed in the experiment is basically a commercially available programmable optical filter (Finisar WaveShaper 4000S) based on liquid crystal on silicon (LCoS) technology. There are four output ports the frequency response (both amplitude and phase) of which can be programmed independently. One or a group (3 comb lines) of the optical tones in channel 1 (Ch1) are fed into a DP-MZM and modulated with a 10 Gbaud QPSK signal. The I and Q signals are generated by a pulse pattern generator (PPG) with $2^{11}-1$ pseudo-random bit sequence (PRBS) patterns. The other optical tone in channel $2(\mathrm{Ch} 2)$ is used as an optical local oscillator (LO) for $\mathrm{THz}$ signal generation. The unmodulated and modulated signals were combined in an optical coupler, aligning their polarizations by using a polarization controller (PC). The optical power of the unmodulated optical tone is controlled by a variable optical attenuator (VOA) to match the 


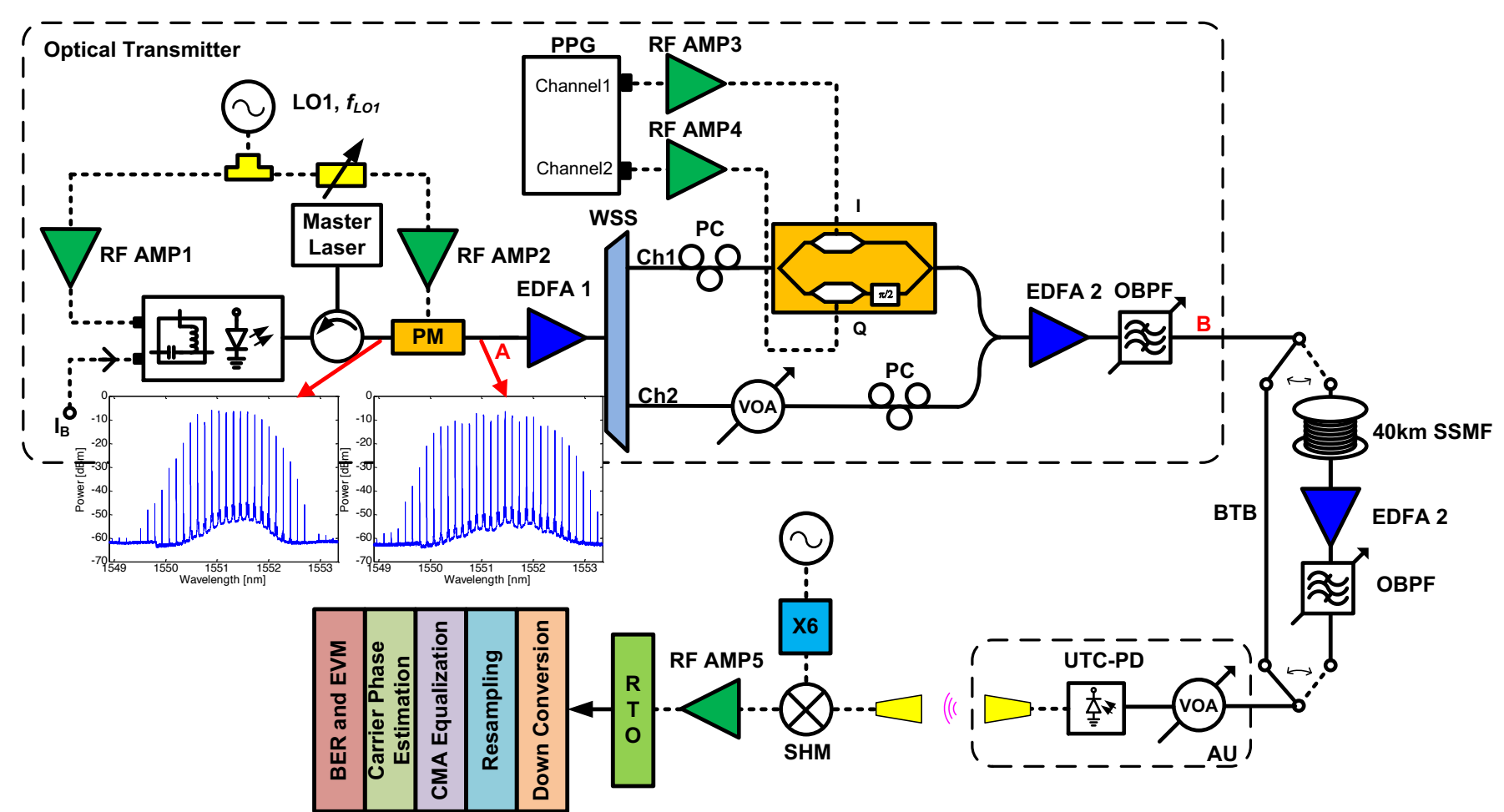

Fig. 6 Experimental setup of the multi-carrier THz system based on a gain-switched laser.

optical power of the other channel. The recombined signal is then amplified with an EDFA and filtered with a $3 \mathrm{~nm}$ optical bandpass filter (OBPF) to reject out-of-band amplified spontaneous emission (ASE). The combined optical signal is transmitted over either a section of back-to-back SSMF or a 40 $\mathrm{km}$ SSMF to the antenna unit (AU). Another EDFA is employed to compensate the loss of the fiber transmission.

At the AU, the optical LO source beats with the modulated optical signal on an unpackaged uni-travelling carrier (UTC) photodiode to generate the $\mathrm{THz}$ modulated multichannel signal [12]. An optical amplifier and VOA were used before the AU to evaluate the system performance. The modulated $\mathrm{THz}$ signal was radiated from the $20 \mathrm{dBi}$ horn antenna and propagated over a $2 \mathrm{~cm}$ wireless channel to a receiving $20 \mathrm{dBi}$ horn antenna. The received $\mathrm{THz}$ signal was initially down-converted to a microwave IF by using a sub-harmonic mixer. The LO signal at $f_{L O 2}$, from a RF synthesizer, is firstly frequency multiplied by using a sixth harmonic electronic multiplier and then applied to the sub-harmonic mixer to down-convert the resultant $\mathrm{THz}$ signal. The down-converted IF signal is then amplified and sent to the real time oscilloscope (RTO) for analog-to-digital conversion. The sampling rate and bandwidth of the RTS are $80 \mathrm{GSample} / \mathrm{s}$ and $36 \mathrm{GHz}$, respectively. An offline digital signal processing (DSP) including downconversion, downsampling, equalization [16], and phase estimation [13] is applied to demodulate the QPSK IF signal using Matlab. It is worthwhile noting that there is no particular frequency offset estimation required in the DSP as the carrier frequency of the $\mathrm{THz}$ signal generated by the beating of the two optical comb lines is very stable.

\section{B. Experimental results}

Fig. 7 shows the optical spectra of the comb (point A in Fig. 6) and the recombined optical tones (point B in Fig. 6). After the photo detection, $170 \mathrm{GHz}, 187 \mathrm{GHz}$ and $204 \mathrm{GHz}$ QPSK signals with a total data rate of $60 \mathrm{~Gb} / \mathrm{s}$ are generated.

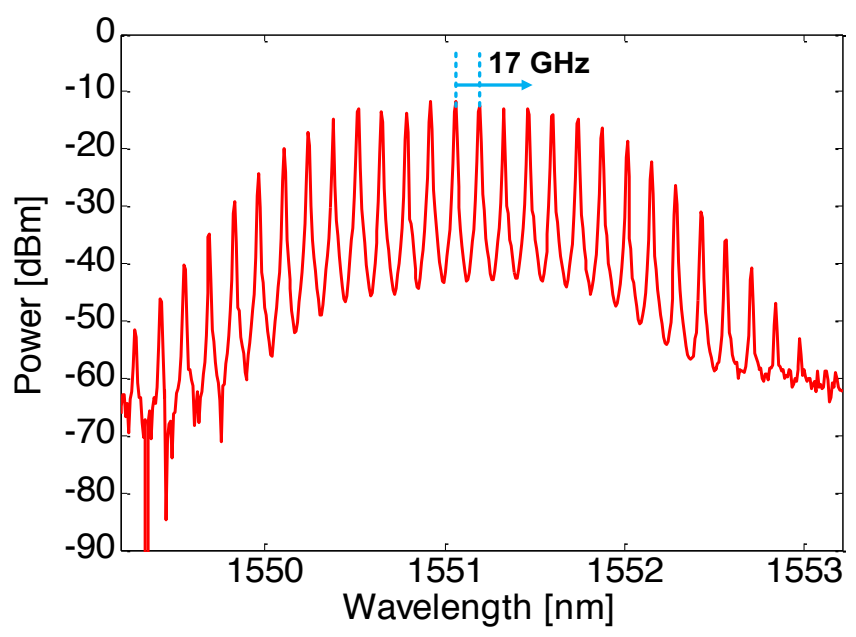

(a) 


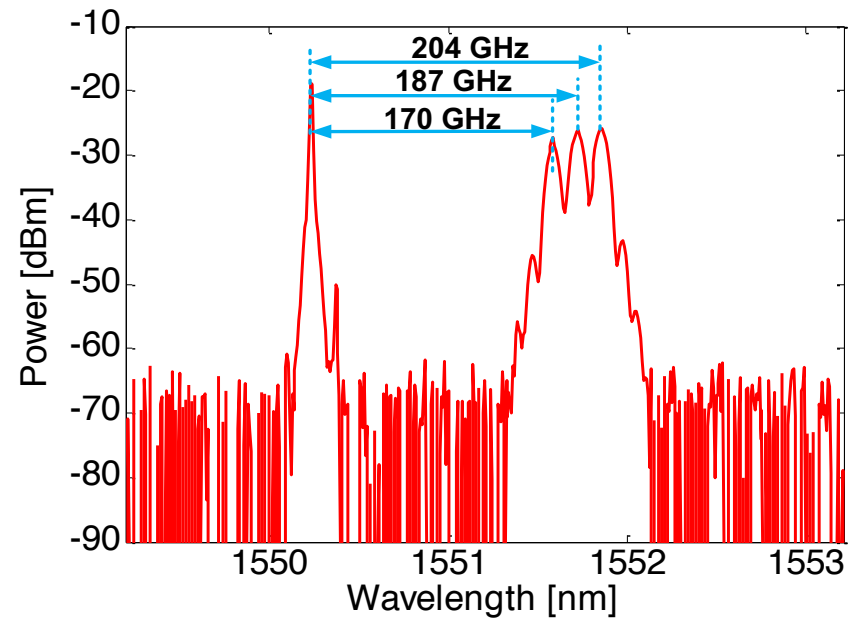

(b)

Fig. 7 Optical spectra of the THz system. (a) optical comb, (b) recombined optical signal. Resolution bandwidth: $0.1 \mathrm{~nm}$.

Fig. 8 (a) and (b) show the EVM and BER as a function of the photocurrent of the UTC-PD respectively. Due to the high phase noise induced by the high-order frequency multiplication, DSP is applied to mitigate the phase noise impact. The optical power into the UTC-PD is varied between 0 to $10 \mathrm{dBm}$. Here we do not show the EVM or BER as a function of input optical power to the UTC-PD, as the photo receiver is not packaged and the optical coupling efficiency is not stable. For experimental simplicity, we only examine the beat of the LO with the central channel (187 GHz signal) as this would suffer the highest cross-talk (worst performing channel) in the system. An EVM as low as $14.7 \%$ is achieved for 3-carrier transmission over $40 \mathrm{~km} \mathrm{SSMF.} \mathrm{It} \mathrm{is} \mathrm{shown} \mathrm{in} \mathrm{Fig.} 8$ that the power penalty induced by the $40 \mathrm{~km}$ SSMF is negligible for single carrier transmission system, while the $40 \mathrm{~km}$ SSMF transmission causes some power penalty in the multi-carrier case, since the fiber chromatic dispersion induced channel decorrelation increased impact of the cross talk.

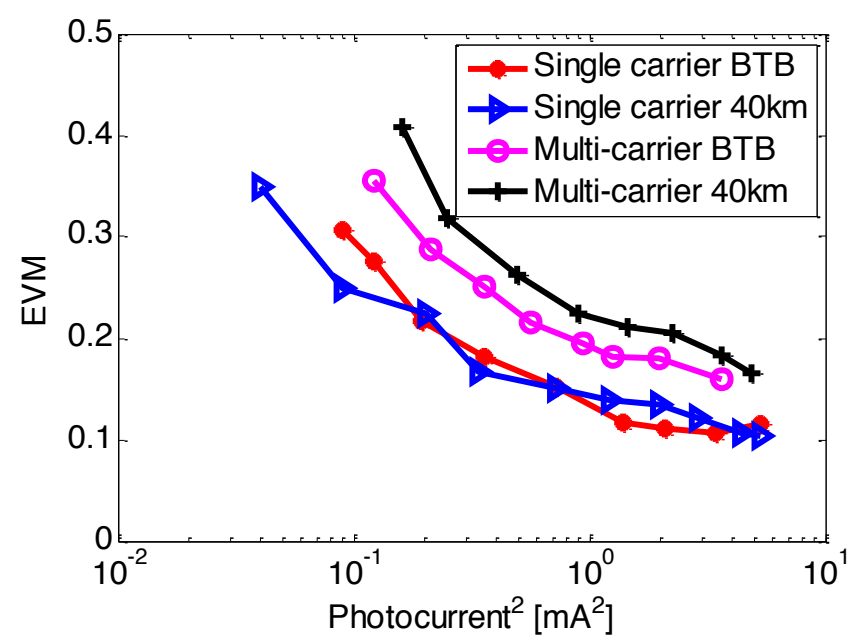

(a)

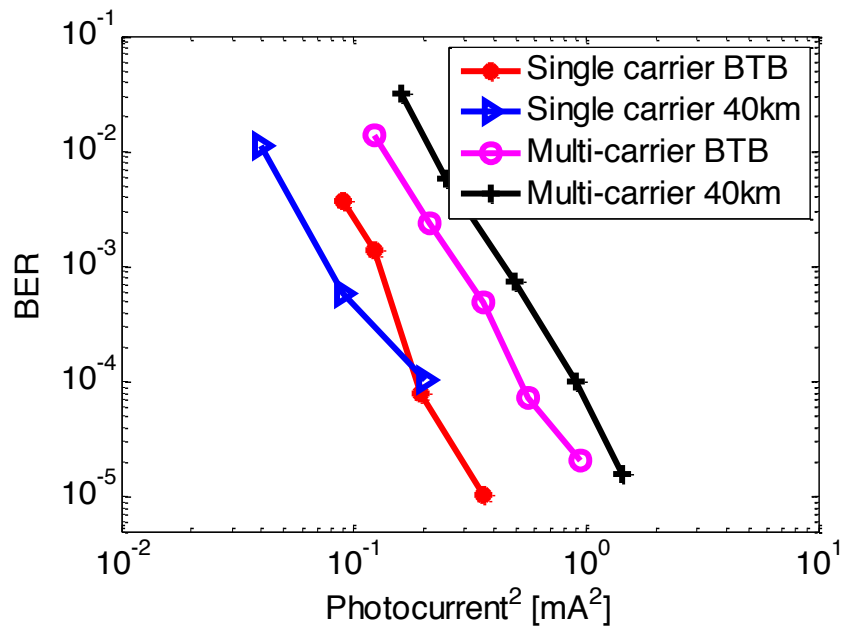

(b)

Fig. 8 EVM and BER as a function of the photocurrent. (a) EVM, (b) BER.

\section{CONCLUSION}

In this paper, we proposed a multi-carrier $\mathrm{THz}$ generation and transmission system employing a gain-switched laser as an optical comb source. The phase noise induced by the higher-order frequency multiplication has been theoretically analyzed and experimentally demonstrated. It has been demonstrated that the phase noise induced by the optical phase decorrelation due to optical delay between the two optical tones can be highly reduced by delay compensation. However the resulting $\mathrm{THz}$ signal may suffer from phase noise due to higher-order frequency multiplication which requires DSP phase correction in the digital transmission system. Furthermore, three 10 Gbaud QPSK THz signal generation and transmission over $40 \mathrm{~km}$ SSMF is experimentally demonstrated, with an EVM as low as $14.7 \%$ achieved for the multi-carrier $\mathrm{THz}$ transmission system. The phase noise impact is highly mitigated by using the DSP.

\section{REFERENCES}

[1]Kleine-Ostmann, and T. Nagatsuma, "A review on terahertz communications research". J. Infrared Millim. Terahertz Waves 32, 143-171 (2011).

[2]A. Seeds, H. Shams, M. Fice, and C. Renaud, "TeraHertz Photonics for Wireless Communications," J. Light. Technol. PP, 1-1 (2014).

[3]X. Pang, et al., "100 Gbit/s hybrid optical fiber-wireless link in the W-band (75-110 GHz)," Opt. Express 19, 24944-9 (2011).

[4]H. Shams, et al., "Photonic generation for multichannel $\mathrm{THz}$ wireless communication," Opt. Express 22, 23465 (2014).

[5]H. Shams, M. J. Fice, K. Balakier, C. C. Renaud, A. J. Seeds, and F. V. Dijk, "Multichannel 200GHz 40Gb / s Wireless Communication System using Photonic Signal Generation," in 2014 International Topical Meeting on Microwave Photonics / The 9th Asia-Pacific Microwave Photonics Conference, 20-23 October, 2014, pp. 4-7.

[6]A. Kanno, K. Inagaki, I. Morohashi, T. Sakamoto, T. Kuri, I. Hosako, T. Kawanishi, Y. Yoshida, and K. Kitayama, "40 Gb/s W-band (75-110 $\mathrm{GHz}$ ) 16-QAM radio-over-fiber signal generation and its wireless transmission," Opt. Express 19, B56-B63 (2011)

[7]T. Nagatsuma, S. Horiguchi, Y. Minamikata, Y. Yoshimizu, S. Hisatake, S. Kuwano, N. Yoshimoto, J. Terada, and H. Takahashi, "Terahertz wireless communications based on photonics technologies," Opt. Express 21, 23736-23747 (2013).

[8]S. Koenig et al. "Wireless sub-THz communication system with high data rate," Nature Photonics, vol. 7, pp. 977-981, 2013. 
[9]P. Anandarajah, R. Zhou, R. Maher, D. G. Pascual, F. Smyth, V. Vujicic and L. Barry, "Flexible Optical Comb Source for Super Channel Systems," in Proc. Optical Fibre Communication Conference, OTh3I.8, March 2013.

[10] T. Shao, M. Beltrán, R. Zhou, P.M. Anandarajah, R. Llorente, and L.P. Barry, "60 GHz radio over fiber system based on gain-switched laser," IEEE/OSA Journal of Lightwave Technology.

[11] T. Shao, E. Martin, P. M. Anandarajah, C. Browning, V. Vujicic, R. Llorente, and L. P. Barry, "Chromatic Dispersion Induced Optical Phase Decorrelation in a $60 \mathrm{GHz}$ OFDM-RoF System," IEEE Photonics Technology Letters.

[12] E. Rouvalis, M. Chtioui, F. van Dijk, F. Lelarge, M. J. Fice, C. C. Renaud, G. Carpintero, and A. J. Seeds, "170 GHz uni-traveling carrier photodiodes for InP-based photonic integrated circuits," Opt. Express, vol 20, no. 18 , pp. $20090-5$, Aug. 2012

[13] A. Leven, N. Kaneda, U.-V. Koc, and Y.-K. Chen, "Frequency Estimation in Intradyne Reception," IEEE Photon.Technol. Lett. 19 (6), 366-368 (2007).

[14] L.S. Cutler, and C.L. Searle, "Some aspects of the theory and measurement of frequency fluctuations in frequency standards," Proceedings of the IEEE, vol. 54, no. 2, 1966, pp. 136-154, 1966.

[15] T. Shao, F. Parésys, G. Maury, Y. Le Guennec, and B. Cabon, "Investigation on the Phase Noise and EVM of Digitally Modulated Millimeter Wave Signal in WDM Optical Heterodyning System," J. Lightwave Technol. 30, 876-885 (2012)

[16] D. L. Jones, "Normalized constant modulus algorithm," in 1995 Conference Record of the Twenty-Ninth Asilomar on Signals, Systems and Computers(1995), pp. 694-697.

Tong Shao received the B. Eng. and M.Eng. degree both from Tsinghua University, Beijing, China, in 2007 and 2009, respectively, and the Ph.D. degree entitled 'Converged $60 \mathrm{GHz}$ Radio over Fiber with WDM-PON Access Networks' from the Institut National Polytechnique de Grenoble (INP-Grenoble), Grenoble, France, in 2012. Between August 2012 to July 2013, he was with the University of Ottawa, as a post-doctoral fellow. He is currently a postdoc researcher in Radio and Optical Communication Lab, Rince Institute, Dublin City University. His research interests include optical communications and radio over fiber.

Haymen Shams received his B.Sc. and M.Sc degrees in electrical and electronic engineering from Alexandria University, Egypt, in 1999 and 2006, respectively and his $\mathrm{PhD}$ degree in electrical engineering from Dublin City University (DCU), Ireland in 2011. His PhD dissertation addressed the optical technologies for generation and distribution of millimetre waves and ultra-wideband RF signals in radio over fibre (RoF) systems. He then worked in photonics group at Tyndall national institute, University College Cork (UCC), Ireland for two years on visible light communication (VLC), and optical coherent receivers. His research interests are on RF-over-fibre for wireless communication including ultra wideband and millimetre wave signals, different optical modulation level formats (such as QPSK, QAM, and CO-OFDM), digital coherent receivers, digital signal processing, optical comb generation, and optical coherent THz. He is currently a research associate in photonic groups, department of electrical and electronic engineering, University College London (UCL).

Dr. Shams is a member of Institute of Electronic and Electrical Engineering (IEEE).

Prince M. Anandarajah received the B.Eng (Electronic Engineering) degree from University of Nigeria, Nsukka in 1992. Subsequently, he worked as an Instructor/Maintenance Engineer at the Nigerian College of Aviation Technology. On completing his M.Eng (1998), he joined the Optical Communications Group at DCU where he obtained his PhD degree (2003). He then worked as a postdoctoral researcher until 2006 and later as a Research Officer with the High Speed Devices and Systems centre which is part of the Rince institute (2007). Currently, he holds a DCU senior research fellow (2011) position. His main research interests include spectrally efficient modulation formats, tunable lasers for re-configurable networks, direct modulation techniques for PONs, generation and optimization of optical frequency combs and short optical pulses and radio-over-fibre distribution systems. He has published over 170 articles in internationally peer reviewed journals and conferences and is also a holder of 4 international patents. He is also a founder and a director of a spin-off company called Pilot Photonics and a senior member of the IEEE.

Martyn J. Fice (S'86-M'87) received the B.A. degree in electrical sciences and Ph.D. degree in microelectronics from the University of Cambridge, Cambridge, U.K., in 1984 and 1989, respectively.

In 1989, he joined STC Technology Laboratories, Harlow, U.K. (later acquired by Nortel), where he was engaged for several years in the design and development of InP-based semiconductor lasers for undersea optical systems and other applications. Subsequent work at Nortel involved research into various aspects of optical communications systems and networks, including wavelength-division multiplexing, all-optical wavelength conversion, optical regeneration, and optical packet switching. In 2005, he joined the Photonics Group, Department of Electronic and Electrical Engineering, University College London, London, U.K., as a Senior Research Fellow. He is now a Lecturer in the same department, with research interests in millimeter and $\mathrm{THz}$ wave generation and detection, optical phase locking, coherent optical detection, optical transmission systems, and photonic integration.

Dr. Fice is a member of the Institution of Engineering and Technology and a Chartered Engineer.

Frédéric van Dijk works at III-V Lab, a joint Laboratory of "Alcatel Lucent Bell Labs", "Thales Research \& Technology" and "CEA-LETI". He is leading the "photonic device for optronics" team involved in design, fabrication and characterisation of optoelectronic devices for microwave and sensing applications. He is in particular studying directly modulated DFB lasers for low loss high dynamic range analog links, mode-locked lasers for telemetry and high speed data sampling, dual wavelength lasers and photonic integrated circuits on InP for microwave to terahertz wave generation.

Alwyn Seeds received the B.Sc, Ph.D. and D.Sc. degrees from the University of London. From 1980 to 1983 he was a Staff Member at Lincoln Laboratory, Massachusetts Institute of Technology, where he worked on GaAs monolithic millimetre-wave integrated circuits for use in phased-array radar. Following three years as lecturer in telecommunications at Queen Mary College, University of London he moved to University College London in 1986, where he is now Professor of Opto-electronics and Head of the Department of Electronic and Electrical Engineering. He has published over 350 papers on microwave and opto-electronic devices and their systems applications. His current research interests include semiconductor opto-electronic devices, wireless and optical communication systems.

Professor Seeds is a Fellow of the Royal Academy of Engineering (UK) and an IEEE Fellow (USA). He has been a Member of the Board of Governors and Vice-President for Technical Affairs of the IEEE Photonics Society (USA). He has served on the programme committees for many international conferences. $\mathrm{He}$ is a co-founder of Zinwave, a manufacturer of wireless over fibre systems. He was awarded the Gabor Medal and Prize of the Institute of Physics in 2012.

Liam P. Barry received the B.E. degree in electronic engineering and the M.Eng.Sc. degree in optical communications from University College Dublin, Dublin, Ireland, in 1991 and 1993, respectively. From 1993 to 1996, he was a Research Engineer with the Department of Optical Systems, France Telecom, Lannion, France, and as a result of this work, he received the Ph.D. degree in optical signal processing from the University of Rennes, France. In 1996, he joined the Applied Optics Centre, University of Auckland, Auckland, New Zealand, as a Research Fellow, where he worked on the use of optical nonlinearities for high speed all-optical switching in fibre networks. In 1998, he was appointed Lecturer with the School of Electronic Engineering, Dublin City University, Dublin, and established the Radio and Optical Communications Laboratory, which is part of the Rince Institute. From 2006 to 2010, he served as a Director of the Rince Institute, an interdisciplinary research centre with over 100 researchers. He is currently a Professor with the School of Electronic Engineering, a Principal Investigator for Science Foundation Ireland, Director of the Radio and Optical Communications Laboratory, and an Associate Dean for Research with the Faculty of Engineering and Computing. His main research interests are all-optical signal processing, optical pulse generation and characterization, hybrid radio/fibre communication systems, and wavelength tunable lasers for reconfigurable optical networks. 